\title{
Automated Generalization and Refinement of Code Templates with Ekeko/X
}

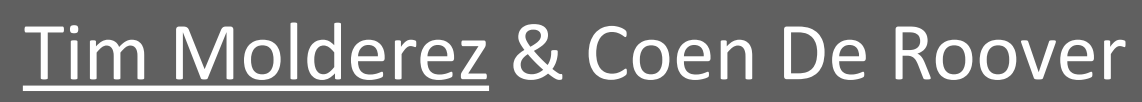




\section{Motivation}

- Code templates lower the learning curve of specifying program searches/transformations

- Hard to master: not always evident to produce only the desired match results 


\section{Ekeko/X}

- Our template-based search/transformation tool

- Search and transform Eclipse Java projects

- Not just syntactic constraints, but semantic ones too 


\section{Ekeko/X}

- Our template-based search/transformation tool

- Search and transform Eclipse Java projects

- Not just syntactic constraints, but semantic ones too

[public void acceptVisitor(ComponentVisitor v) ...] @[(invoked-by ?x)]

[....acceptVisitor(...)]@[(equals ?x)] 


\section{Ekeko/X}

- Our template-based search/transformation tool

- Search and transform Eclipse Java projects

- Not just syntactic constraints, but semantic ones too

[public void acceptVisitor(ComponentVisitor v) ...] @[(invoked-by ?x)]

[....acceptVisitor(...)]@[(equals ?x)] 


\section{Ekeko/X}

- Our template-based search/transformation tool

- Search and transform Eclipse Java projects

- Not just syntactic constraints, but semantic ones too

[public void acceptVisitor(ComponentVisitor $v$ ) ...] @[(invoked-by ?x)]

[....acceptVisitor(...)]@[(equals ?x)] 


\section{How to create this template}

public void acceptVisitor(ComponentVisitor $v$ ) \{

Iterator $<$ Component $>i=e$ lements. iterator () ; while $(i \cdot \operatorname{hasNext}())\{$

Component comp $=($ Component $) \mathrm{i} \cdot \operatorname{next}()$;

comp.acceptVisitor $(v)$;

\}

\}

cs.acceptVisitor(vstor); 


\section{How to create this template}

public void acceptVisitor(...) ...

comp.acceptvisitor(v);

Too few matches! 


\section{How to create this template}

public void acceptVisitor(...) ...

....acceptvisitor (...);

Too many matches! 


\section{Recommending changes}

- How to assist users if a template produces too few/many matches?

- Genetic search approach: Find a sequence of mutations leading to more desired matches

- User marks what is desired/undesired 


\section{Genetic algorithm}

$$
\text { बहो }
$$

Initial template 


\section{Genetic algorithm}

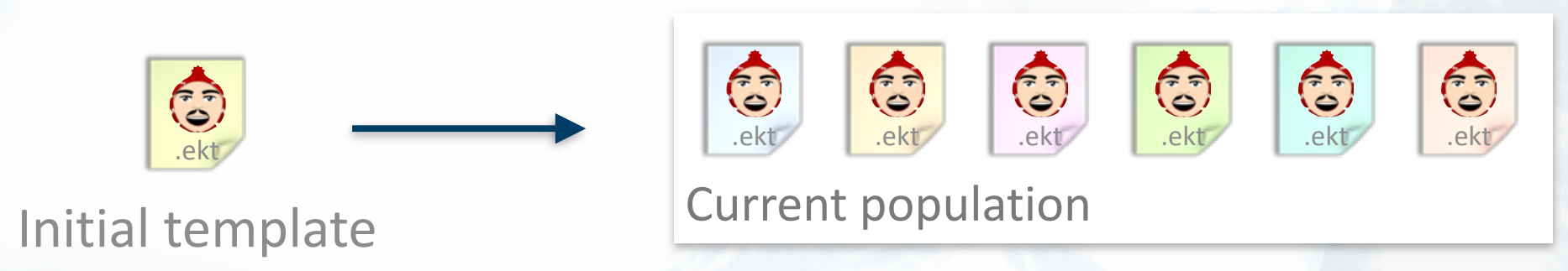




\section{Genetic algorithm}

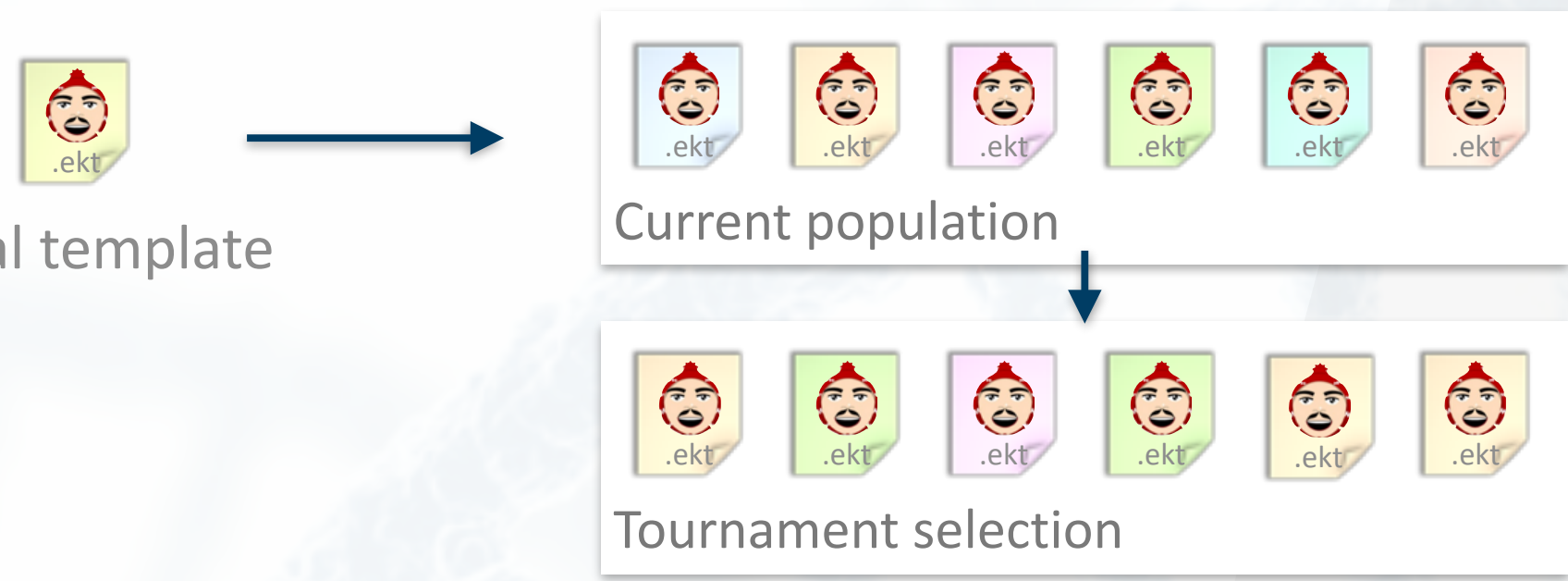




\section{Genetic algorithm}
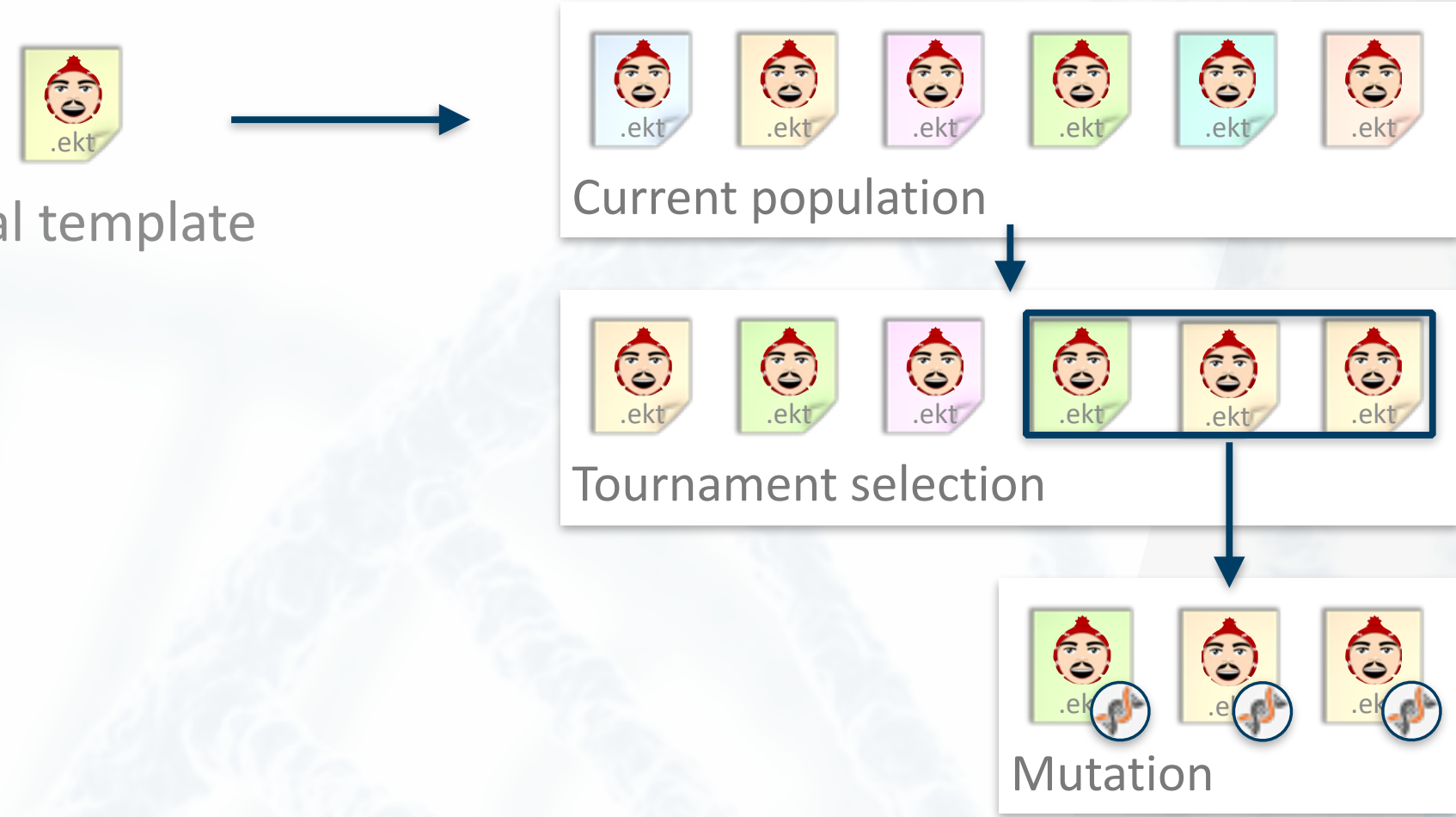


\section{Genetic algorithm}

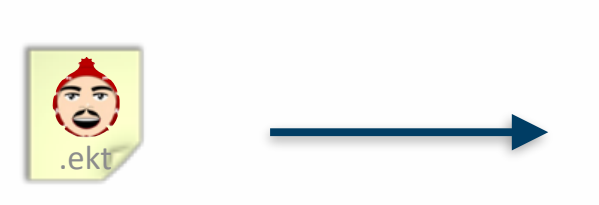

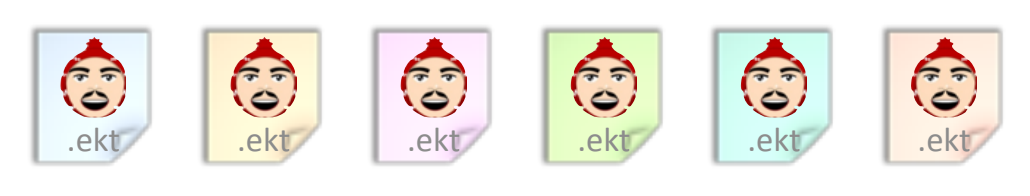

Initial template

\section{Current population}

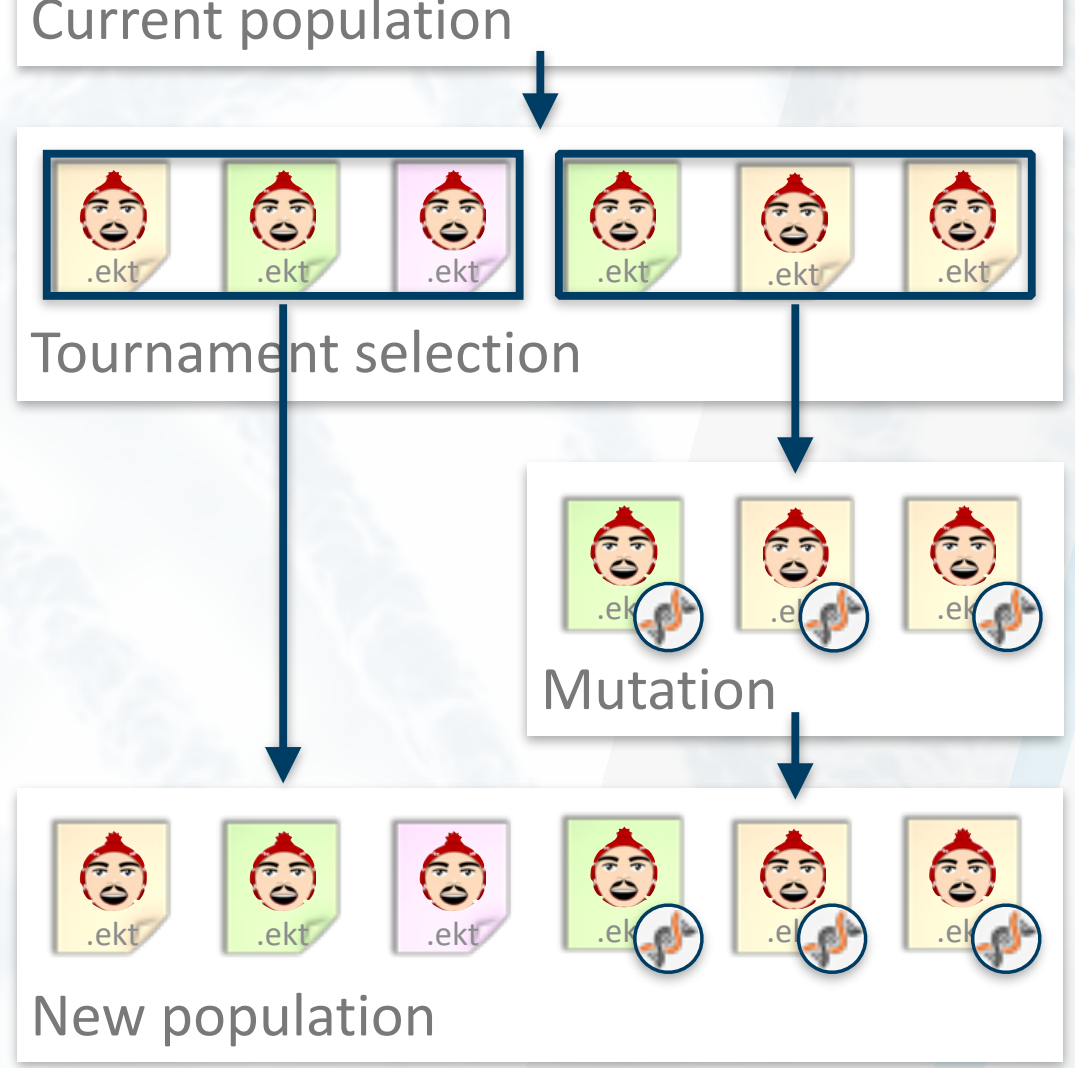




\section{Genetic algorithm}
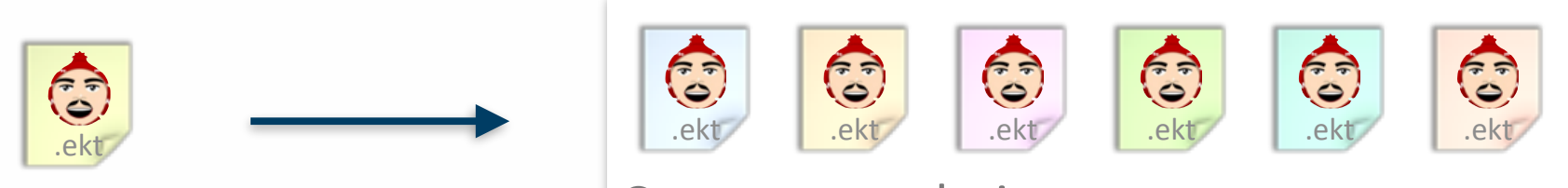

Initial template

\section{Current population}
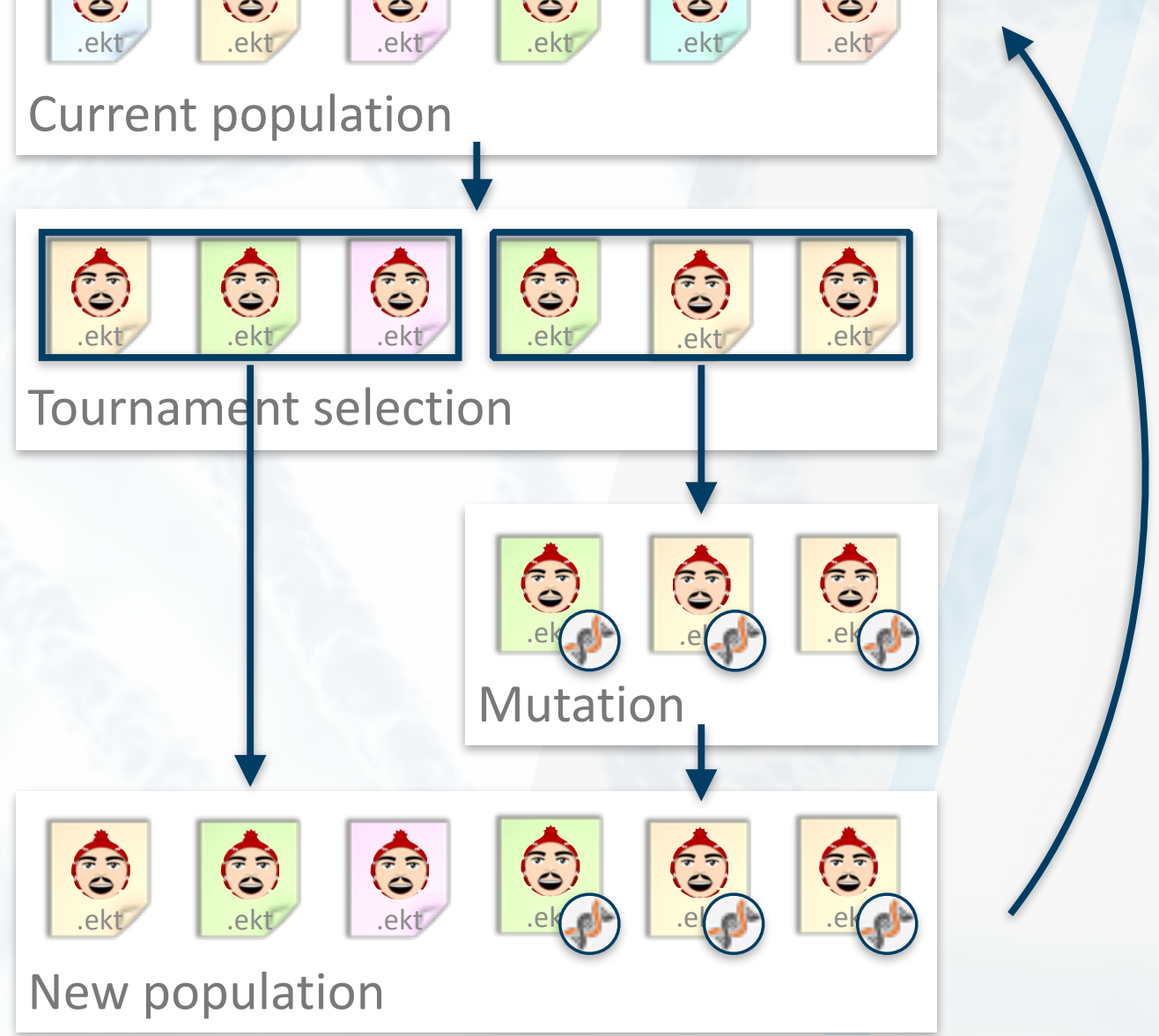


\section{Genetic algorithm}

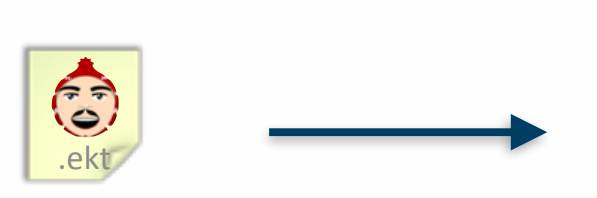

Initial template

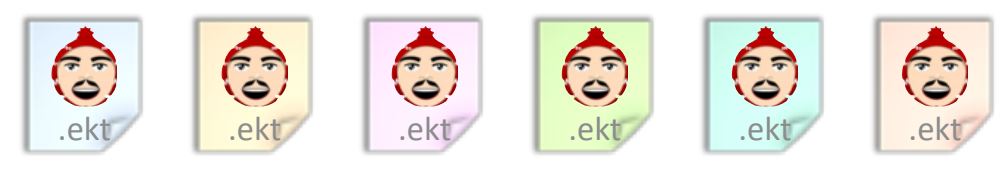

Current population
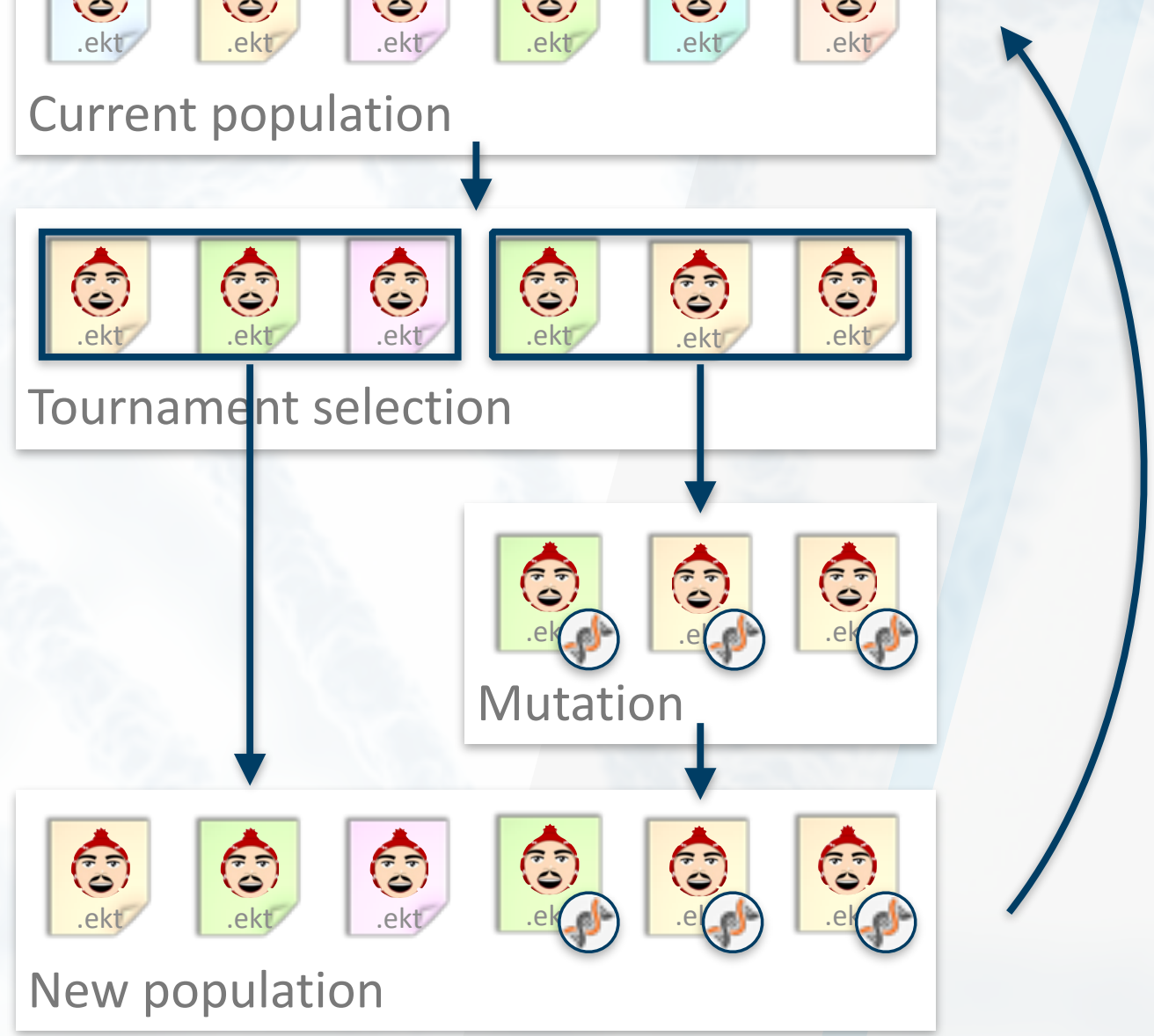

Solution template 
Fitness function 


\section{Fitness function}

- Number between 0 and 1 , based on two components: 


\section{Fitness function}

- Number between 0 and 1 , based on two components:

- F-score: how many (un)desired matches?

- In terms of true/false pos. \& false neg.

- Coarse-grained 


\section{Fitness function}

- Number between 0 and 1 , based on two components:

- F-score: how many (un)desired matches?

- In terms of true/false pos. \& false neg.

- Coarse-grained

- Partial score:

- For each desired snippet, how close is the template to finding it?

- Fine-grained 
Demo 


\section{Summary}

- Assist Ekeko/X users by means of a genetic search algorithm that can recommend template mutations

- Future work: Towards transformations

- Find Ekeko/X on Github:

github.com/cderoove/damp.ekeko.snippets

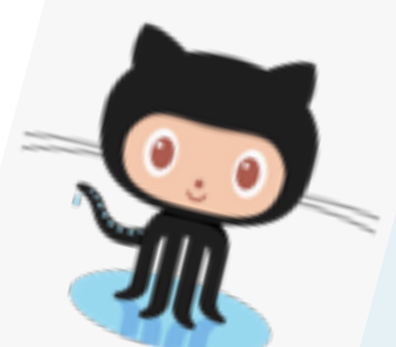

